\title{
Characterisation of Ionic and Lipid Gradients within Corpus Callosum White Matter after Diffuse Traumatic Brain Injury in the Rat
}

\author{
David Hartnell, ${ }^{\mathrm{a}, \mathrm{b}}$ Kate Gillespie-Jones, ${ }^{\mathrm{c}}$ Cristina Ciornei, ${ }^{\mathrm{c}}$ Ashley Hollings, ${ }^{\mathrm{a}, \mathrm{b}}$ Alexander Thomas, ${ }^{\mathrm{a}, \mathrm{b}}$ \\ Elizabeth Harrild, ${ }^{\mathrm{a}, \mathrm{b}}$ Juliane Reinhardt,,${ }^{\mathrm{d}, \mathrm{e}}$ David J Paterson, ${ }^{\mathrm{d}}$ Dasuni Alwis, ${ }^{\mathrm{c}}$ Ramesh Rajan, ${ }^{\mathrm{c}}$ Mark J \\ Hackett ${ }^{\mathrm{a}, \mathrm{b} *}$ \\ ${ }^{a}$ Curtin University, Curtin Institute for Functional Molecules and Interfaces, School of Molecular and Life \\ Sciences, Perth, WA, AUS 6845 \\ ${ }^{\mathrm{b}}$ Curtin University Curtin Health Innovation Research Institute, Perth, AUS 6102 \\ ${ }^{c}$ Monash University, Neuroscience Program, Biomedicine Discovery Institute and Department of Physiology, \\ Clayton, VIC, AUS 3168 \\ d Australian Nuclear Science and Technology Organisation, 800 Blackburn Road, Clayton, VIC, AUS 3168 \\ e ARC Centre of Excellence for Advanced Molecular Imaging, La Trobe University, Institute for Molecular \\ Sciences, Department of Chemistry and Physics, Melbourne, VIC, AUS 3086
}

\begin{abstract}
There is increased recognition of the effects of diffuse traumatic brain injury (dTBI), which can initiate yet unknown biochemical cascades, resulting in delayed secondary brain degeneration and long term neurological sequela. There is limited availability of therapies that minimise the effect of secondary brain damage on the quality of life of people who have suffered TBI, many of which were otherwise healthy adults. Understanding the cascade of biochemical events initiated in specific brain regions in the acute phase of dTBI, and how this spreads into adjacent brain structures may provide the necessary insight to drive development of improved therapies. In this study we have used direct biochemical imaging techniques (Fourier transform infrared spectroscopic imaging) and elemental mapping (X-ray fluorescence microscopy) to characterise biochemical and elemental alterations that occur in corpus callosum white matter in the acute phase of dTBI. The results provide direct visualisation of differential biochemical and ionic changes that occur in the highly vulnerable medial corpus callosum white matter relative to the less vulnerable lateral regions of the corpus callosum. Specifically, the results suggest that altered ionic gradients manifest within mechanically damaged medial corpus callosum, potentially spreading to and inducing lipid alterations to white matter structures in lateral brain regions.
\end{abstract}

\section{KEY WORDS}

Brain injury, Ions, White Matter, X-ray fluorescence, Synchrotron, Infrared

\section{INTRODUCTION}

Traumatic brain injury is one of the largest causes of morbidity in otherwise healthy adults. ${ }^{1-4}$ The incidence of traumatic brain injury across adult populations typically shows a peak amongst adult populations $18-25$ years in age (attributed to increased risk taking actions), with a secondary increase in incidence in elderly populations (attributed to increased incidence of falls) ${ }^{5-10}$ In severe cases TBI is fatal but even if people survive TBI, a substantial proportion suffer long term cognitive, learning and memory deficits associated with secondary brain damage. ${ }^{2,11,12}$ There is therefore, a strong drive to characterise and understand the chemical alterations that occur early after TBI, i.e. in the acute phase, which may initiate or drive mechanisms of secondary brain damage and contributing to long term neurological sequelae. $^{13}$ 
The major form of TBI is diffuse TBI (dTBI), where there is no direct damage by a foreign object to the brain, but the impact-induced relative motion between skull and brain tissue $^{14}$ results in shear forces that cause axonal swelling, tearing and disconnection, ${ }^{10,15,16}$ and even synaptic shearing. ${ }^{17}$ A significant amount of work on dTBI has identified many of the secondary damage processes in dTBI, of which a focal point of change is in the blood-brain barrier, as well as oedema, inflammation and vascular changes, and network degeneration, ${ }^{13}$ and we have identified the consequent immediate and medium-term effects on cortical neuronal responses as a suppression that extends through the cortical column. ${ }^{18,19}$ With the exception of one study on mechanic injury, ${ }^{20}$ nothing is known about the effects of any form of TBI on the changes in distribution and concentration of elements such as $\mathrm{P}, \mathrm{S}, \mathrm{Cl}, \mathrm{K}, \mathrm{Ca}, \mathrm{Fe}, \mathrm{Mn}, \mathrm{Cu}$. All of the above elements however, have been shown to be altered in a range of brain neurodegenerative disorders and in ageing, ${ }^{21-25}$ with implications for a range of cellular biochemical reactions in which these elements play a role in the normal brain and in neurodegeneration. It is hoped that increased understanding of elemental alterations that occur in vulnerable and less vulnerable brain regions, in the acute phase of TBI, may provide insight into the cascade of biochemical pathways that may be activated and subsequently contribute to delayed degeneration and cognitive impairment in mid- and chronic phases after TBI.

Recent advances in synchrotron x-ray fluorescence microscopy (XFM) enable rapid elemental mapping of brain tissue sections, at micron spatial resolution, to study the distribution and concentration of elements like those noted above. ${ }^{26-29}$ The advantages of "multi-modal" imaging are also gaining increasing recognition, 30,31 where vibrational spectroscopic methods such as Fourier transform infrared spectroscopy (FTIR), or Raman spectroscopy are applied to characterise tissue biochemistry in association with elemental distributions revealed by XFM. ${ }^{25}, 29,32-34$ FTIR-imaging is increasingly used as an analytical tool to investigate holistic biochemical composition at the cellular (i.e. micron spatial resolution) or near-cellular (i.e. $10-50 \mu \mathrm{m}$ spatial resolution) level. This sort of multi-modal approach has been used to associate lipid homeostasis with ionic or elemental gradients after ischemic, ${ }^{29,}{ }^{34}$ or haemorrhagic stroke, ${ }^{35,}{ }^{36}$ or to associate protein aggregation with brain metal content during dementia or Alzheimer's disease. ${ }^{33,37,38}$

In this study we have applied a multi-modal approach using FTIR biochemical imaging and XFM elemental mapping to characterise biochemical alterations, ionic gradients, and transition metal content in the medial and lateral regions of the corpus callosum white matter of rodents, and surrounding brain structures, following dTBI, created using a well-established and characterised model of diffuse TBI (dTBI) in rodents, which model clinical aspects of human brain injury, particularly to white matter structures such as the corpus callosum. ${ }^{39-41}$ The findings lay an important framework for future studies to use direct spectroscopic imaging methods, in addition to classical biochemical methods, to investigate and visualise the timeline and cellular location in which ionic gradients are disturbed following dTBI. Such information may provide vital insight into the initial cellular processes that are affected in the acute phase of dTBI, which may subsequently drive secondary degeneration.

\section{RESULTS \& DISCUSSION}

FTIR imaging reveals differential lipid changes across medial and lateral regions of the corpus callosum after dTBI.

In this study, FTIR-imaging was conducted at near-cellular spatial resolution $(25 \mu \mathrm{m})$ to assess the holistic biochemical composition of medial and lateral regions of the corpus 
callosum following dTBI (Figure 1 and Figure 2). Specifically, FTIR-imaging was used to compare lipid composition of the lateral and medial regions of the corpus callosum, between sham and dTBI animals, using spectroscopic markers of lipid methylene groups $v_{\mathrm{s}}\left(\mathrm{CH}_{2}\right)$ (Figure 1A, E), lipid esters $v(\mathrm{C}=\mathrm{O})$ (Figure 1B, F), and phosphate head group of phospholipids $v_{\text {as }}\left(\mathrm{PO}_{2}{ }^{-}\right)$(Figure 1C, F). A representative FTIR spectrum that highlights the location of these spectroscopic marker bands is presented in Figure 2A. Statistical analysis with a two-way ANOVA revealed a strong interaction effect $(p<0.0001)$ for all three lipid markers, between anatomical region (medial vs lateral) and treatment group (sham vs TBI). Post-hoc testing of multiple comparisons (Sidak's method) revealed that dTBI has a differential effect on lipid composition of medial relative to lateral corpus callosum white matter. Specifically, the spectroscopic markers of lipids, lipid methylene groups (Figure 1I), lipid esters (Figure 1J), and phosphate head groups (Figure $1 \mathrm{~K}$ ) were observed to decrease in medial corpus callosum of dTBI animals relative to sham animals. This result indicates lipid damage and lipid loss within the medial corpus callosum, which was expected, and is consistent with the established vulnerability of the medial corpus callosum to mechanical damage in this model. ${ }^{41-44}$

To further confirm that the loss of lipids from the medial corpus callosum after dTBI is due to phospholipid damage, spectroscopic alterations to the phosphate functional groups were investigated in greater detail. Spectra were vector normalised to the phosphate band (1200 $1250 \mathrm{~cm}^{-1}$, to remove variation associated with absolute changes in lipid content. Analysis of second-derivative spectra was then performed to identify if chemical alterations to the phosphate group were present. Close inspection of the FTIR spectra in the $v_{\text {as }}\left(\mathrm{PO}_{2}^{-}\right)$region indicated reduced intensity at $1223 \mathrm{~cm}^{-1}$ in the medial corpus callosum of dTBI rats (Figure $1 \mathrm{D}, 1 \mathrm{H}$ and $2 \mathrm{~B}$ ). Statistical analysis with a two-way ANOVA revealed a strong interaction effect between the factors of anatomical location and treatment group $(p=0.0006)$. Post-hoc multi-comparison testing (Sidak's method) confirmed that a statistically significant reduction in hydrated phospholipid head groups is observed in the medial regions of the corpus callosum, but not the lateral regions of the corpus callosum (Figure 2L). This spectroscopic alteration is consistent with reduction in the content of hydrated phospholipids, which absorb at this spectral location. ${ }^{45}$ Although multiple organo-phosphate compounds absorb infrared light across the $1200-1250 \mathrm{~cm}^{-1}$ range (e.g., ATP, phosphocreatine, RNA, and DNA), ${ }^{45}$ the primary absorbing species in white matter is likely to be phospholipids due to the low cell density and high myelin content of this region. The observation of spectroscopic markers of phospholipid degradation within medial corpus callosum white matter after dTBI that have been observed in this study, is consistent with the studies of others, which used biochemical assays to demonstrate phospholipid degradation as a hall mark of acute TBI pathology. ${ }^{46-49}$ Indeed, quantification of phospholipid breakdown products, such as glycerol, in the interstitial space are commonly used markers of TBI severity. ${ }^{47,48}$

Spectroscopic markers of phospholipid degradation were only observed in the medial region of the corpus callosum, and not the lateral regions in this study, suggesting that the medial corpus callosum white matter has heightened vulnerability to mechanical damage induced by shock waves during dTBI ${ }^{50}$ Intriguingly, a significant increase in lipid methylene, lipid ester, and phospholipid headgroup content was observed in the lateral corpus callosum of dTBI rats relative to sham animals (Figure 1). This observed differential effect of dTBI on lipid composition of medial and lateral corpus callosum white matter supports the existence of differential biochemical responses to dTBI across the brain. ${ }^{41,43}$

To further investigate the myelin content of the corpus callosum, immuno-fluorescence (IF) measurement of myelin basic protein antigenicity and luxol fast blue (LFB) myelin histochemistry was performed (Figure 3). LFB staining (Figure 3A-D) revealed reduced 
average myelin content within the medial corpus callosum of dTBI animals relative to sham, however, the difference was not statistically significant (Figure 3I). No differences in LFB staining were observed between the lateral regions of the corpus callosum of dTBI animals relative to sham (Figure 3I). Similarly, no difference in MBP antigenicity measured by IF (Figure 3E-H) was observed between dTBI and sham animals in either the medial or lateral regions of the corpus callosum (Figure $3 \mathrm{~J}$ ). It is possible that the lack of statistically significant differences could be the result of the sample preparation methods used in this study. Flashfrozen non-fixed tissue was essential for FTIR and XFM measurements, and therefore, tissue sections had to be post-fixed in formalin after being sectioned, for LFB and IF analyses. Such protocols are not optimal for IF and histochemistry (perfusion fixation would be preferred), and may have contributed to increased variance in the data set. Alternatively, the results may reflect that LFB and MBP-IF inherently detect proteins in the myelin sheath. LFB is widely regarded to stain lipo-proteins, and MBP-IF is protein specific. Therefore, degradation of phospholipids from the myelin sheath that occurs without substantial protein loss, would be expected to result in biochemical differences in the corpus callosum that are detected by FTIR, but not LFB or MBP-IF. In addition, alterations in cellular lipid levels will be detected by FTIR, but will not be detected by LFB or MBP-IF.

At this stage the exact reasons for the increase in lipid content within the lateral regions of the corpus callosum are not known, however, altered oligodendrocyte metabolism or abnormal myelination may account for the observation. Studies by others have shown that abnormal and often extended myelin wrapping occurs as early as 2 days post traumatic axonal injury, ${ }^{51,52}$ and increased abundance of excessively myelinated axons has been observed following axonal trauma. ${ }^{53}$ Likewise, increased abundance of myelinating cells has been reported within the corpus callosum in the acute phase following TBI ${ }^{54}$ In particular, the increased appearance of myelinating cells have been proposed to originate from the subventricular zone of the lateral ventricles, which is immediately adjacent to the lateral corpus callosum. ${ }^{54}$ Therefore, although degradation of the myelin sheath is a hall mark of axonal injury, our findings raise the possibility of increased myelin production in adjacent, less damaged regions. Although our results did not directly show increased myelin, as measured by MBP-IF or LFB, the increased lipid content may be the initial stages of lipid synthesis and accumulation within oligodendrocytes. If so, it is still not known if such processes are the initial stages of remyelination or, if the net lipid increase reflects abnormal myelination and oligodendrocyte dysfunction. If the latter, this would be likely contribute to the extended, incorrectly folded myelin layers observed through electron microscopy in other studies. ${ }^{51,52}$ FTIR imaging studies by others have revealed increased lipid levels in brain cells associated with inflammation, such as during chronic pain. ${ }^{55}$ The elevated lipid content observed in the lateral corpus collosum following dTBI in this study may therefore, possibly be a marker of inflammation, which has been shown to occur in this model. ${ }^{41}$

XFM elemental mapping reveals effects of dTBI on the levels of metal ions and ionic gradients across lateral and medial corpus callosum white matter

XFM elemental mapping was used to assess metal ion ( $\mathrm{Fe}, \mathrm{Cu}, \mathrm{Zn})$ and diffusible ion $\left(\mathrm{Cl}^{-}, \mathrm{K}^{+}, \mathrm{Ca}^{2+}\right)$ gradients across the corpus callosum white matter. The results demonstrate TBI has a significant effect on $\mathrm{Zn}$ concentration in the corpus callosum ( $\mathrm{p}=0.0015$, two-way ANOVA, Figure 4A-D, M)), but not Fe or Cu (Figure 5D, E). Sidak post-hoc testing of multicomparisons identified that the medial sub-region of the corpus callosum contains less $\mathrm{Zn}$ in TBI animals relative to shams (Figure 4M), however, no significant difference between TBI and sham was observed in the lateral region (Figure 4M). This finding is consistent with the established heightened vulnerability of the medial corpus callosum to mechanical damage, 
relative to the lateral sub-region. The exact mechanism of $\mathrm{Zn}$ loss from the medial corpus callosum is not known. However, the myelin sheath has numerous $\mathrm{Zn}$ binding sites, at which $\mathrm{Zn}$ binding is proposed to help stabilise myelin structure and maintain integrity of the myelin sheath wrapping axons. ${ }^{57}$ Myelin damage or an altered chemical environment within myelin during pathology may be expected to decrease white matter $\mathrm{Zn}$ content. Therefore, preferential loss of $\mathrm{Zn}$ from the medial corpus callosum, taken together with the lipid loss observed from FTIR data, is interpreted to reflect axonal damage after dTBI.

In addition to transition metal ions ( $\mathrm{Fe}, \mathrm{Cu}, \mathrm{Zn}$ ), XFM elemental mapping was used to semi-quantitatively image the distribution of diffusible ions $\mathrm{Cl}^{-}, \mathrm{K}^{+}$, and $\mathrm{Ca}^{2+}$. TBI was found to not have an effect on the areal density of the ions $\mathrm{Cl}^{-}, \mathrm{K}^{+}$, and $\mathrm{Ca}^{2+}$, in the corpus callosum (summarised in Figure 5A-C). On average the $\mathrm{Cl}^{-}$concentration was higher in the corpus callosum of dTBI rats relative to sham rats, while the $\mathrm{K}^{+}$were lower on average. To decrease external sources of variance, such as tissue thickness, a $\mathrm{Cl}^{-} / \mathrm{K}^{+}$ration was calculate. Statistical analysis with a two-way ANOVA revealed an effect of TBI on the $\mathrm{Cl}^{-} / \mathrm{K}^{+}$ratio in the corpus callosum (Figure 4E-H). Post-hoc testing of multiple comparisons revealed that the $\mathrm{Cl}^{-} / \mathrm{K}^{+}$ratio was significantly elevated in the medial region of the corpus callosum of TBI animals, relative to sham animals (Figure 4N). The elevation of the $\mathrm{Cl}^{-} / \mathrm{K}^{+}$ratio was found to be trending towards significance in the lateral region of the corpus callosum in dTBI animals, relative to shams $(p$ $=0.056$ ). The $\mathrm{Cl}^{-} / \mathrm{K}^{+}$ratio is of specific physiological importance in tissues, especially white matter, as it reflects that ability of white matter glial (astrocytes and oligodendrocytes) to regulate ionic gradients. ${ }^{58-61}$ It has been established that elevation of the $\mathrm{Cl}^{-} / \mathrm{K}^{+}$ratio occurs after specific physiological events such as ischemia, hypoxia, edema, and is a common marker of pathology. ${ }^{58-61}$

Ionic imbalances are associated with impaired volume regulation, which negatively impacts on the ability of astrocytes and oligodendrocytes to support axons, and therefore, impacts brain signalling capacity). Maintaining ionic gradients in the white matter is vital for maintenance of axon-glial connections, and oligodendrocyte function. ${ }^{58-61}$ At this stage, we can only speculate on the origin and consequence of altered $\mathrm{Cl}^{-} / \mathrm{K}^{+}$ratio observed in the corpus callosum white matter. However, the data is consistent with early mechanical damage to the vulnerable medial corpus callosum white matter (also evidenced by loss of lipids and $\mathrm{Zn}$ ), which then may propagates ionic disturbances throughout the corpus callosum. The pattern of the $\mathrm{Cl}^{-} / \mathrm{K}^{+}$ratio in the lateral corpus callosum is visually consistent with a "spreading wave" of altered ionic gradients (Figure 4), even though the average ratio did not reach statistical significance $(p=0.056)$. A physiological event such as a spreading wave of altered ionic gradients may account for the differential lipid response observed between the medial and lateral regions of the corpus callosum. Specifically, lipid loss occurs in medial corpus callosum associated with mechanical damage but, altered ionic gradients that spread into the lateral corpus callosum, where there is not severe mechanical damage, may trigger altered glial function and increased myelin synthesis. This study has not examined markers of brain inflammation, as we have examined animals at 24 hours post injury, while inflammatory markers (e.g., astrogliosis, increased abundance of activated microglia) are not typically observed until beyond 24 hours post injury. ${ }^{68-70}$ Therefore, it is unlikely that brain inflammation is a key driver of the observations of this study, but rather, the altered ionic gradients and lipid damaged detected may play a role in propagating brain inflammation in the mid- and chronic periods post TBI. ${ }^{68-72}$ In particular, glia and micro-glia not only regulate extra-cellular ionic concentrations, but react to altered extra-cellular environments and a spreading wave of altered $\mathrm{Cl}^{-} / \mathrm{K}^{+}$ratio as indicted by this study may contribute to brain inflammation. To investigate this further, future studies are now clearly required to investigate the finer timeline of phospholipid degradation, lipid alterations, and altered ionic balance in medial and lateral regions of the 
corpus callosum and the surrounding hippocampal and cortical structures following dTBI, especially with respect to markers of brain inflammation and secondary tissue damage.

\section{CONCLUSION}

In conclusion, this paper has demonstrated a differential ionic and biochemical response to dTBI across the medial and lateral corpus callosum. Spectroscopic markers characteristic of phospholipid damage and disturbed ionic gradients were observed in the medial region of the corpus callosum, which is known to be most vulnerable to mechanical trauma in this model. Our approach of utilising multi-modal imaging was the key to visualise altered ionic gradients that possibly extend to the lateral regions of the corpus callosum, where spectroscopic markers of heightened lipid synthesis, but not phospholipid damage were observed. This was the first study to identify a potential link between altered ionic gradients and lipid metabolism following dTBI, but such a finding is not without support in the literature. In fact, the results from this study help support the findings of others that have identified changes to oligodendrocyte function and abnormal myelin wrapping following traumatic injury to the brain.

The collective findings of this study suggests that disturbed ionic gradients that propagate from the site of mechanical damage after dTBI, may contribute to altered glial function and ensuing biochemical changes. Future studies are required and will focus on spatiotemporal characterisation of the ionic changes that follow dTBI, with a specific focus on their effect on astrocyte and oligodendrocyte function.

\section{METHODS}

\section{Animal Model -dTBI}

Male Sprague Dawley rats aged between 10-12 weeks were obtained from Monash Animal Services and allowed to acclimatize in local holding facility for 1 week prior to surgery. During this period rats were housed in $12 \mathrm{hr}$ light/dark cycle with food and water ab libitum and closely monitored to anticipate the ideal time for surgery when animals weigh between 340-360 grams. Rats were then randomly allocated to either a Sham-control $(n=6)$ or dTBI $(n$ =6) animal group.

To create diffuse TBI, a modified version of the WDAI model was used, ${ }^{40}$ in which a metal disc is fixed to the skull to prevent skull fracture and focal lesions. Anaesthesia was induced with $5 \%$ isoflurane in a mixture of $20 \%$ oxygen $/ 80 \%$ nitrogen. Noxious hindlimb pinching and the absence of palpebral reflexes were used to determine adequate anaesthesia. Once this was obtained animals were intubated with $16 \mathrm{G}$ intravenous catheter (Introcan safety 16G, B. Braun medical Inc., Bethlehem, PA, USA) and ventilated with $3 \%$ isoflurane in $20 \%$ oxygen $/ 80 \%$ nitrogen at 80 breaths per minute using a mechanical ventilator (Ugo, Basile ventilator, 7026). Body temperature was maintained $37-38^{\circ} \mathrm{C}$ by placing rats on a thermostatically controlled heat pad. The scalp was shaved and a midline incision approximately $2 \mathrm{~cm}$ in length made to expose the skull. The metal disk $(1 \mathrm{~cm}$ in diameter and $3 \mathrm{~mm}$ thick) was then fixed to skull between bregma and lambda. The animal was then placed on a foam bed and body secured with masking tape to allow rapid acceleration and deceleration of the head. A 450gram weight guided by a plexiglass tube was dropped from a height of 2 metres directly onto the metal disc to cause moderate-severe injury. The animal was then rapidly reconnected to the ventilator carrying $20 \%$ oxygen $/ 80 \%$ nitrogen in the absence of 
anaesthetic. The animal was monitored until spontaneous breathing resumed which usually occurred within 10 minutes. During ventilation the metal disk was removed, and skull checked for fractures. If skull fractures were detected the animal was immediately euthanized. If there were no fractures present the incision was sutured and cleaned with betadine. For sham controls the same protocol was followed minus the traumatic impact. Rats were placed back into individual houses and positioned on a heat pad. Recovery was monitored at regular intervals and rats were allowed free access to water and food. 24 hours post-surgery, to produce diffuse TBI or sham animals, the animals were euthanized with Sodium pentobarbital (Lethabarb, Virbac, Australia) and brains rapidly removed. All experimental procedures were conducted in accordance with Monash University Standing Committee on Ethics in Animal Experimentation.

\section{Animal Model - Lipid Profiles in Nä̈ve Corpus Callosum}

Brain tissue was harvested from naïve wild type C57 BL/6 mice $(n=6)$ and SpragueDawley rats $(n=6)$. Animals were housed in ordinary cages in a colony room on a 12 hour light/dark cycle at a controlled temperature $\left(21^{\circ} \mathrm{C}\right)$ with normal rodent maintenance water and chow available ad libitum. All experimental procedures were conducted in accordance with Curtin University Animal Ethics Guidelines and the Monash Animal Ethics Committee.

\section{Sample Preparation}

For all samples, brain tissue was rapidly dissected and flash frozen in liquid nitrogen to preserve the in vivo biochemical and elemental content, and to avoid introduction of chemical artefacts that can occur during chemical fixation, as previously shown. ${ }^{62},{ }^{63}$ Serial $10-\mu \mathrm{m}$-thick coronal brain sections were cut with a cryo-microtome at $-18{ }^{\circ} \mathrm{C}$ and melted onto: a silicon nitride substrate $\left(5 \times 5 \mathrm{~mm}^{2}\right.$ and $1 \mu \mathrm{m}$ thick membrane, Melbourne Centre for Nanofabrication) for XFM analyses; $1 \mathrm{~mm}$ thick CaF2 substrate (Crystran) for FTIR analyses, and superfrost plus glass microscope slides for routine H\&E histology, luxol fast blue histochemistry, or myelin basic protein immuno fluorescence.

\section{Identification of Regions of Interest}

Representative examples of the regions of interest analysed in this study are presented in Figure 6. Identification of the regions of interest was achieved with elemental maps ( $\mathrm{K}$ or $\mathrm{Zn}$ ) and FTIR images of lipid distribution, as these elemental and biochemical markers provide simple recognition of anatomical brain structures. Confirmation of regions of interest was confirmed with H\&E histology of adjacent tissue sections, as well as with comparison to white matter distribution revealed by luxol fast blue histochemistry. It should be noted that the spatial resolution of XFM $(\sim 1-2 \mu \mathrm{m})$ and FTIR $(25 \mu \mathrm{m})$ is substantially poorer than optical microscopy $(\sim 0.5 \mu \mathrm{m})$. Therefore, it is still possible that the lateral corpus callosum ROIs contain possible input from the cortico-thalamic fiber bundles, which we can not exclude at the spatial resolution of our imaging techniques. We have performed analysis at naïve rats and mice that demonstrate the biochemical composition of the white matter tracks varies as a function of anatomical location (Supporting Information Figure 1), and this is an important consideration for interpretation of our results.

\section{FTIR Spectroscopic Analyses}

FTIR spectroscopic images of brain tissue sections were collected with at $25 \mu \mathrm{m}$ spatial resolution using Nicolet iN 10MX FTIR microscope, with an 8x2 pixel liquid nitrogen cooled linear array detector and $25 \mu \mathrm{m}$ pixel size, as previously described. ${ }^{25}$ Spectra were collected at 
$4 \mathrm{~cm}^{-1}$ spectral resolution with 16 co-added scans, and the background was collected using the same parameters from a blank $\mathrm{CaF}_{2}$ substrate. One of the individual pixels on the linear array detector displays reduced sensitivity, resulting in increased noise that can be seen as a stripe artefact in some images. These pixels were excluded from all ROI's, so they did not affect statistical analyses.

FTIR spectroscopic images were analysed with Cytospec v2.00.03 and OPUS v7.0. Second-derivative spectra were calculated from vector normalized raw spectra (vector normalized to the amide I band, $1700-1600 \mathrm{~cm}^{-1}$ ), using a 9 smoothing point Savitzky-Golay function, for analysis of aggregated protein content. Lipid homeostasis was determined from characteristic spectroscopic markers of lipid ester and lipid methylene groups, which were generated from the area under the curve of the ester carbonyl band $\left(1755-1715 \mathrm{~cm}^{-1}\right)$ and lipid methylene groups $v_{\mathrm{s}}(\mathrm{CH} 2)\left(2865-2840 \mathrm{~cm}^{-1}\right)$, using a linear background subtraction across the same spectral range, in non-normalised and non-derivatised spectra. Phospholipids were evaluated from the area under the curve of the $v_{\mathrm{as}}\left(\mathrm{PO}_{2}^{-}\right)\left(1250-1200 \mathrm{~cm}^{-1}\right)$. Second-derivative analysis of the $v_{\mathrm{as}}\left(\mathrm{PO}_{2}^{-}\right)$band (vector normalised from $1250-1200 \mathrm{~cm}^{-1}$ ) was used to differentiate between hydrogen bonded and non-hydrogen bonded phospholipids, as described in the literature. ${ }^{45,64}$ For statistical analysis, regions of interest were determined for the medial and lateral corpus callosum and the average area under the peak or second-derivative intensity calculated for the spectroscopic markers described above.

\section{Synchrotron X-ray Fluorescence Microscopy}

Elemental mapping of tissue sections was performed at the X-ray Fluorescence Microscopy beamline at the Australian Synchrotron, as previously described. ${ }^{25,28,37}$ In brief, a monochromatic X-ray beam with energy $15.8 \mathrm{keV}$ was focused to a $\sim 1 \mu \mathrm{m}$ (1-sigma) spot, using a Kirkpatrick-Baez mirror pair. The X-ray emissions from the sample mounted perpendicular to the beam were collected in event-mode using the low-latency, 384-channel Maia detector in backscatter geometry. ${ }^{65,}{ }^{66}$ The sample was raster scanned through the beam with an effective dwell time of $0.1 \mathrm{~ms}$ per effective step (image pixel) size of $1 \mu \mathrm{m}$, and images were processed with a 3 point moving average to increase signal to noise and enhance image contrast, as previously described. ${ }^{25,28,37}$ Elemental foils (Micromatter, Canada), were scanned in the same geometry, as references for elemental quantification. Elemental maps of areal density were reconstructed from the full emission spectra with GeoPIXE v6.6j (CSIRO, Australia), using a linear transformation matrix for spectral deconvolution. The composition of the tissue sample was approximated as dried organic material $\left(\mathrm{C}_{22} \mathrm{H}_{10} \mathrm{~N}_{2} \mathrm{O}_{4}\right)$, with a density of $1.42 \mathrm{~g} \mathrm{~cm}^{-3}$, as in previous studies. ${ }^{25,37,63,67}$ Quantitative elemental maps were extracted as TIFF files of per-pixel elemental areal density in $\mathrm{ng} \mathrm{cm}^{-2}$, which were then imported into ImageJ v1.48.

\section{H\&E Histology, Luxol Fast Blue Histochemistry, and Myelin Basic Protein Immuno- Fluorescence Microscopy}

$H \& E$ histology: Tissue cryo-sections were air-dried and post-fixed in $10 \%$ buffered formalin (Sigma) for haematoxylin and eosin (H\&E) staining. Comparison of histology images with elemental maps and FTIR spectroscopic images was used to determine regions of interest corresponding to medial and lateral corpus callosum, and surrounding brain structures such as the cortex and hippocampus. Microscopy images of H\&E stained tissue were collected at 4x magnification using a Olympus Bx51 microscope with Olympus dp70 camera and cellSans Standard software. 
LFB-histochemistry: Tissue sections were fixed post tissue sectioning using $1 \mathrm{~mL}$ of $10 \%$ buffered formalin dropped on top of the tissue section. After fixation, slides were rinsed in phosphate buffered saline (PBS, $10 \mathrm{mM}$ ). Tissue sections were then defatted in a 1:1 alcohol:chloroform mixture for 2 hours. Tissue sections were rehydrated back to $95 \%$ ethanol, before incubation in luxol fast blue (Sigma Aldrich) at $56{ }^{\circ} \mathrm{C}$, overnight. Following incubation, tissue sections were rinsed in distilled water and differentiated in $\mathrm{Li}_{2} \mathrm{CO}_{3}$. Slides were then rinsed in a series of distilled water, $95 \%$ ethanol, 100\% ethanol, Xylene, and then coverslipped. Microscopy images of LFB stained tissue were collected at $4 \mathrm{x}$ magnification using a Olympus Bx51 microscope with Olympus dp70 camera and cellSans Standard software. Images were saved as grey-scale tiffs, for semi-quantitative analysis of myelin.

MBP-IF: Myelin basic protein (MBP) immuno-fluorescence was performed using rabbit anti-MBP (Abcam, ab40390, Cambridge, USA). Tissue sections were fixed post sectioning using $1 \mathrm{~mL}$ of $10 \%$ buffered formalin dropped on top of the tissue section. After fixation, slides were rinsed in phosphate buffered saline (PBS, $10 \mathrm{mM}$ ), and blocked in bovine serum albumin for 1 hour at room temperature. Following blocking, the tissue sections were incubated overnight in primary antibody (concentration 1:200) at $4{ }^{\circ} \mathrm{C}$. Following incubation with primary anti-body, slides were rinsed in n phosphate buffered saline (PBS, $10 \mathrm{mM}$ ), and incubated with the secondary antibody (goat anti-rabbit IgG Alex Fuor@488) at a concentration of 1:200, for 2 hours at room temperature. Lastly, the slides were rinsed with PBS, mounted with aqueous antifade medium,coverslipped, and stored at $-20^{\circ} \mathrm{C}$. Fluorescence microscopy images were collected at 4x magnification using a Olympus Bx51 microscope with Olympus dp70 camera and cellSans Standard software, using blue light excitation and collection of emitted fluorescence using a green filter. Images were saved as grey-scale tiffs for semi-quantitative analysis of myelin basic protein.

\section{Statistical Analysis}

All data is presented as the group mean $\pm \sigma$ (standard deviation) and therefore, the distance between the minimum error bar and the maximum error bar is equal to $2 \sigma$. The experimental design of this investigation has incorporated 2 independent variables, animal model (dTBI or sham) and anatomical brain region (medial or lateral corpus callosum). The medial and lateral regions of the corpus callosum that were investigated are schematically shown in Figure 6. The main effects of the two independent variables on the dependent variable (elemental or biochemical content) was evaluated using a two-way ANOVA (GraphPad Prism v7.04). Post-hoc testing of multiple comparisons was performed using a Sidak test, $(95 \%$ confidence limit). Post-hoc testing was only performed if the two-way ANOVA revealed a statistically significant main effect of the animal model, or an interaction effect. All experimental animal groups contain $n=6$ animal replicates for FTIR or XFM analyses. experimental groups contained $n=5$ animal replicates for LFB and MBP-IF analyses. 


\section{FIGURES}

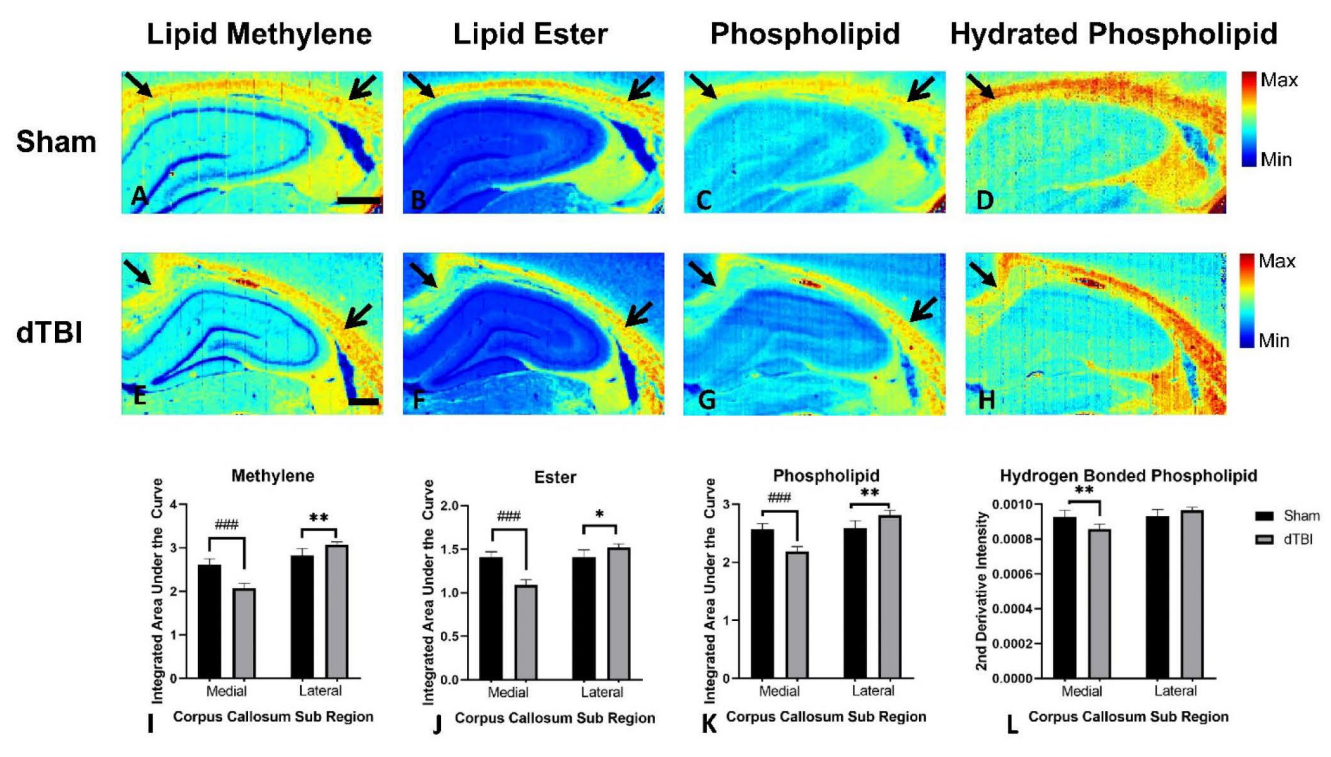

Figure 1: FTIR spectroscopic imaging of medial and lateral corpus callosum lipid composition after dTBI. The integrated area under the curve was used to assess relative content of lipid methylene (A, E), lipid esters $(\mathbf{B}, \mathbf{F})$, and phosphate head groups of lipids $(\mathbf{C}, \mathbf{G})$. The falsecolour FTIR functional group images reveal decreased content of all three lipid markers in medial corpus callosum following dTBI (E-H), relative to sham (A-D). The decreased content is visualised through greater abundance of light blue or dark blue pixels in medial corpus callosum (closed arrow heads) of dTBI images relative to sham. Increased content of all three lipid markers was observed in lateral dTBI corpus callosum as observed by greater abundance of dark red pixels in dTBI images relative to sham (open arrow heads). Assessment of the chemical environment of the phosphate head group of phospholipids $(\mathbf{D}, \mathbf{H})$ revealed decreased relative abundance of hydrated phospholipids in medial corpus callosum (closed arrow) in dTBI animals (light blue pixels, H) compared to sham animals (yellow/red pixels, D). Scale bars $=500 \mu \mathrm{m}$. Min - Max values for FTIR images are as follows: 0 to 4 (Lipid Methylene), 0 to 2 (Lipid Ester), 0 to 2 (Phospholipid), -0.001 to 0 (Hydrated Phospholipid).

The visual observations represented in the false-colour FTIR functional group images were confirmed with a two-way ANOVA and post-hoc multiple comparison testing. $* p<0.05, * *$ $p<0.01, * * * p<0.001$, \#\#\# $p<0.0001$ 

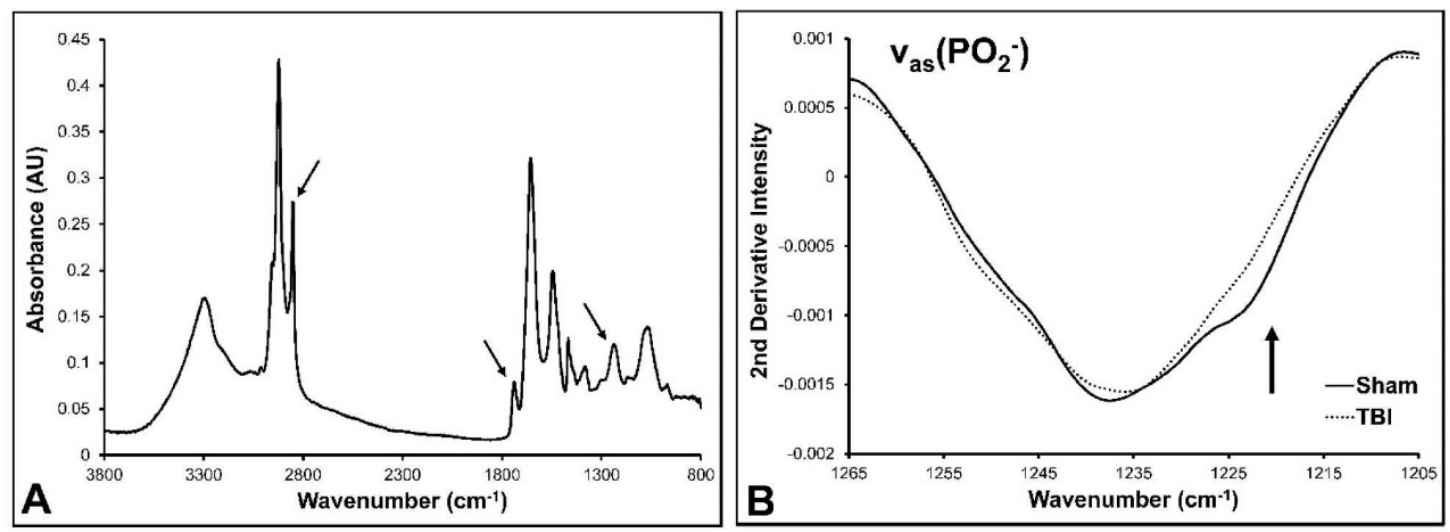

Figure 2: Representative FTIR spectra (A) showing spectroscopic marker bands (arrows) of lipid methylene groups $v_{\mathrm{s}}\left(\mathrm{CH}_{2}\right)$ at $2852 \mathrm{~cm}^{-1}$, lipid esters $v(\mathrm{C}=\mathrm{O})$ at $1745 \mathrm{~cm}^{-1}$, and phosphate head group $v_{\text {as }}\left(\mathrm{PO}_{2}^{-}\right)$at $1235 \mathrm{~cm}^{-1}$. The second-derivative FTIR spectrum $(\mathbf{B})$ highlights the decreased intensity at $1223 \mathrm{~cm}^{-1}$ in representative spectra from the medial corpus callosum of a dTBI animal, relative to a sham animal, indicative of decreased hydrated phospholipids following dTBI. Note: second-derivative inverts the direction of peaks, relative to original spectrum.
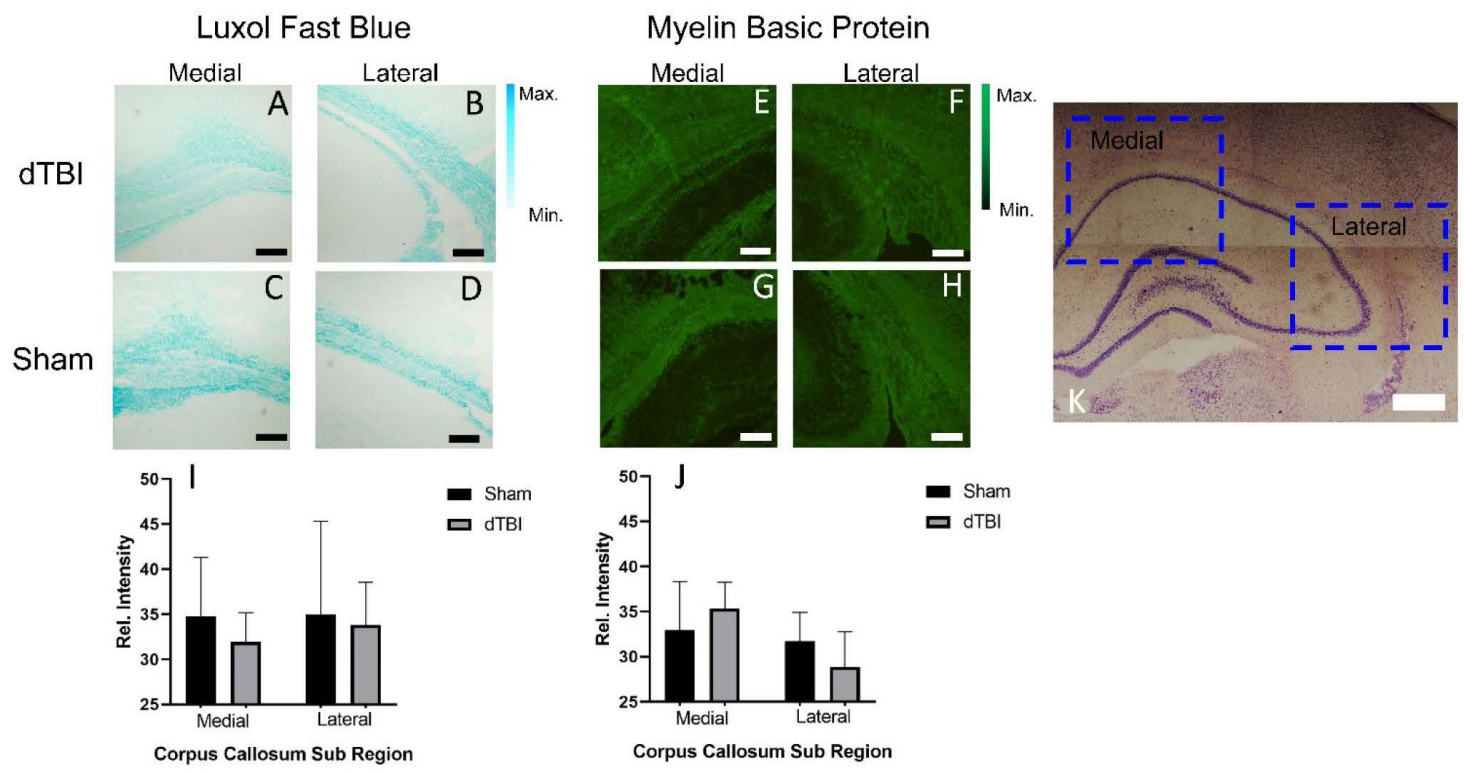

Figure 3: Assessment of myelin with luxol fast blue (LFB) histochemistry (A-D) and myelin basic protein (MBP) immuno-fluorescence (IF) (E-H), in medial corpus callosum $(\mathbf{A}, \mathbf{C}, \mathbf{E}, \mathbf{G})$ and lateral corpus callosum $(\mathbf{B}, \mathbf{D}, \mathbf{F}, \mathbf{H})$, in sham animals $(\mathbf{C}, \mathbf{D}, \mathbf{G}, \mathbf{H})$ and dTBI animals $(\mathbf{A}$, $\mathbf{B}, \mathbf{E}, \mathbf{F})$. Statistical analysis (two-way ANOVA, $n=5$ in sham and $n=5$ in dTBI groups)) did not reveal statistical differences in myelin density as studied by LFB histochemistry (I) or MBP-IF (J), however, on average LFB staining was reduced in medial corpus callosum of dTBI animals relative to shams (I). H\&E histology (K) shows the anatomical locations (blue dash boxes) of medial and lateral tissue shown in panels A-H. Scale bar $(\mathrm{A}-\mathrm{H})=200 \mu \mathrm{m},(\mathrm{K})$ $=500 \mu \mathrm{m}$. 

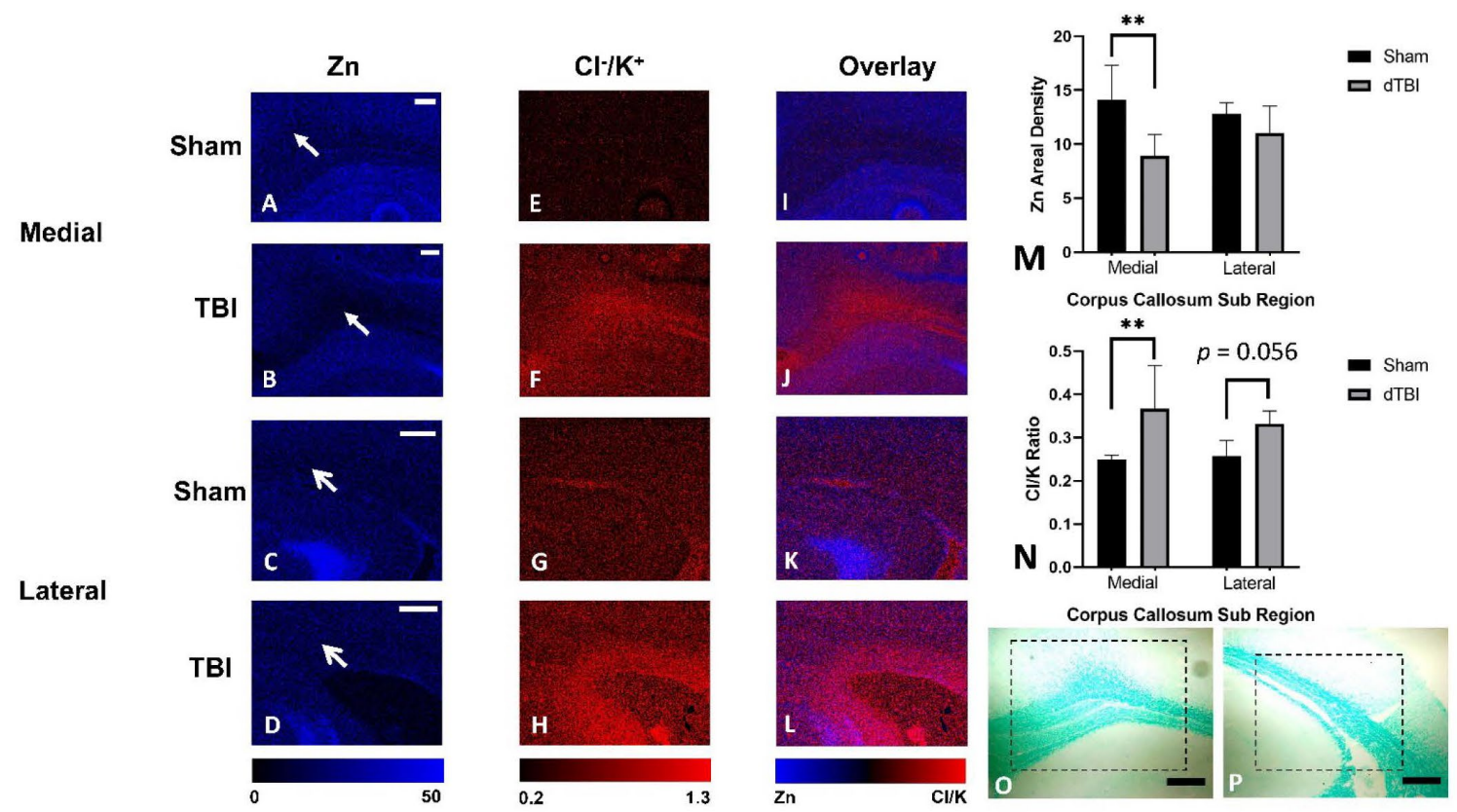

Figure 4: XFM elemental mapping reveals (A-D) $\mathrm{Zn}$ areal density $\left(\mathrm{ng} \mathrm{cm}^{-2}\right)$ is decreased in medial corpus callosum (closed arrow heads) after dTBI relative to shams, but $\mathrm{Zn}$ is not decreased in lateral corpus callosum (open arrow heads). The $\mathrm{Cl}^{-} / \mathrm{K}^{+}$ratio (E-H) was observed to be elevated in both medial and lateral corpus callosum after dTBI. Overlay of the $\mathrm{Zn}$ and $\mathrm{Cl}^{-}$ $/ \mathrm{K}^{+}$ratio is also shown $(\mathbf{I}-\mathbf{L})$. The visual observations of representative images were confirmed with two-way ANOVA statistical analyses of $\mathrm{Zn}(\mathbf{M})$ and $\mathrm{Cl}^{-} / \mathrm{K}^{+}$ratio $(\mathbf{N})$, and post-hoc multiple comparison testing (Sidak's test). No significant differences were observed for other elements $(\mathrm{Cl}, \mathrm{K}, \mathrm{Ca}, \mathrm{Cu}, \mathrm{Fe})$, which are presented in Figure 5. The approximate anatomical locations for the XFM elemental mapping are shown on LFB stained sections, for medial corpus callosum $(\mathbf{O})$ and lateral corpus callosum $(\mathbf{P})$. Scale bar $=200 \mu \mathrm{m} .{ }^{*} p<0.05,{ }^{* *} p<$ 0.01 .
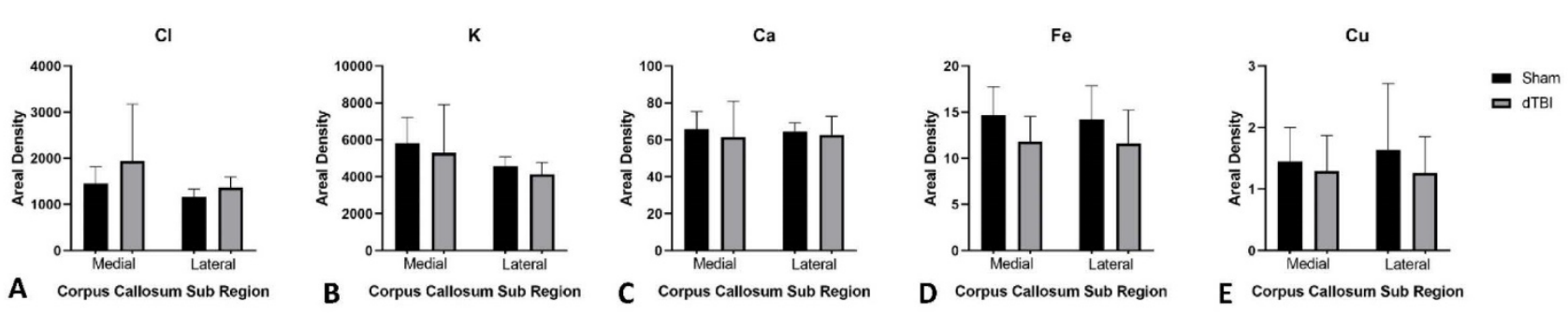

Figure 5: Summary of XFM elemental content in medial and lateral corpus callosum, which were not significantly effected by dTBI $\left(\mathrm{Cl}^{-}, \mathrm{K}^{+}, \mathrm{Ca}^{2+}, \mathrm{Cu}, \mathrm{Fe}\right)$, shown in panels A-E, respectively. No significant difference was observed with two-way ANOVA statistical analyses. 
H\&E

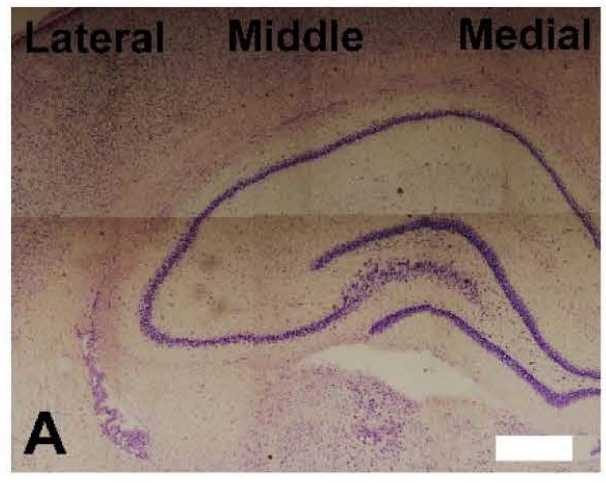

FTIR (Lipid)

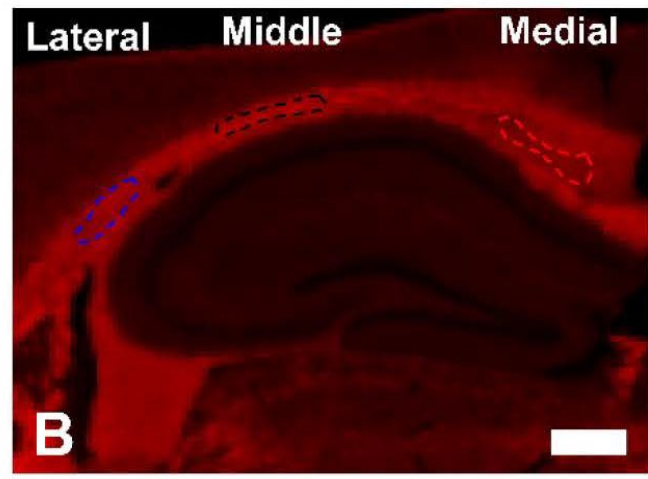

Figure 6: Anatomical regions of interest within corpus callosum were guided by histology (A). An example of the lateral (blue dashes), middle (black dashes), and medial (red dashes) regions of interest are shown annotated onto a monochrome FTIR image of lipid distribution in representative sham animal (B). In dTBI study lateral and medial corpus callosum was compared. Naïve lipid distribution was compared in mice and rats across medial, middle, and lateral regions. Scale bar $=500 \mu \mathrm{m}$.

\section{SUPPORTING INFORMATION}

SI Figure 1 shows lipid distribution in corpus callosum in naïve mice and rats.

SI Figure 2 shows MBI immuno-histochemistry positive and negative controls.

\section{AUTHOR INFORMATION}

Corresponding author: Dr Mark J Hackett

Curtin Institute for Functional Molecules and Interfaces, School of Molecular and Life Science, Curtin University, Perth, WA 6845, AUS

Email: mark.j.hackett@curtin.edu.au

\section{AUTHOR CONTRIBUTIONS}

$\mathrm{DH}$ (PhD Student) completed majority of data analysis and major student contributor to manuscript writing.

KGJ(PhD student) Ran dTBI model and generated brain tissue samples.

CC (Research Associate) Assisted with dTBI model and generated brain tissue samples, and provided guidance for sample preparation, histology, and histochemistry.

AT (Undergraduate student) Performed pilot experiments for DH, preliminary FTIR and histology characterisation of dTBI tissue samples.

AH (PhD student) Prepared tissue sections and collected FTIR data for naïve rat brain analysis

EH (Undergraduate student) Prepared tissue sections and collected FTIR data for naïve mouse brain analysis

JR (Beamline Scientist) Assisted in XFM data collection and data analysis 
DP (Beamline Scientist) Assisted in XFM data collection and data analysis, and provided training for XFM data analysis.

DA (Research Fellow) Supervised and provided training for generation of dTBI tissue samples. Developed experimental design and conceptual ideas in collaboration with RR and MJH. Provided expertise on dTBI.

RR (Co-Investigator) Developed experimental design and conceptual ideas in collaboration with DA and MJH. Provided expertise in brain anatomy and dTBI. Primary supervisor of KGJ.

MJH (Corresponding Author), Supervisor of DH, AH, EH. Developed experimental design and conceptual ideas in collaboration with DA and RR. Provided expertise for multimodal FTIR and XFM mapping.

All authors were involved in critically assessing data and deriving scientific conclusions. All authors were involved in manuscript editing.

\section{ACKNOWLEDGEMENTS}

We gratefully acknowledge travel funding provided by ANSTO, funded by the Australian Government. Components of this research were undertaken at the X-ray fluorescence Microscopy (XFM) beamline at the Australian Synchrotron, ANSTO, Victoria, Australia. This work was performed in part at the Melbourne Centre for Nanofabrication (MCN) in the Victorian Node of the Australian National Fabrication Facility (ANFF). RR acknowledges funding support from the National Health and Medical Research Council NMHRC APP 1029311. MJH is supported by the Dementia Australia Research Foundation, Mamutil New Investigator Project Grant (11646).

\section{REFERENCES}

1. Hyder, A. A., Wunderlich, C. A., Puvanachandra, P., Gururaj, G., and Kobusingye, O. C. (2007) The impact of traumatic brain injuries: a global perspective, NeuroRehabilitation 22, 341-353.

2. Roozenbeek, B., Maas, A. I., and Menon, D. K. (2013) Changing patterns in the epidemiology of traumatic brain injury, Nat. Rev. Neurol. 9, 231-236.

3. Langlois, J. A., Rutland-Brown, W., and Wald, M. M. (2006) The epidemiology and impact of traumatic brain injury: a brief overview, J. Head Trauma Rehabil. 21, 375-378.

4. Hyder, A. A., Wunderlich, C. A., Puvanachandra, P., Gururaj, G., and Kobusingye, O. C. (2007) The impact of traumatic brain injuries: a global perspective, NeuroRehabilitation 22, 341-353.

5. Narayan, R. K., Michel, M. E., Ansell, B., Baethmann, A., Biegon, A., Bracken, M. B., Bullock, M. R., Choi, S. C., Clifton, G. L., and Contant, C. F. (2002) Clinical trials in head injury, J. Neurotrauma 19, 503-557.

6. Myburgh, J. A., Cooper, D. J., Finfer, S. R., Venkatesh, B., Jones, D., Higgins, A., Bishop, N., and Higlett, T. (2008) Epidemiology and 12-month outcomes from traumatic brain injury in Australia and New Zealand, J. Trauma Acute Care Surg. 64, 854-862.

7. Park, E., Bell, J. D., and Baker, A. J. (2008) Traumatic brain injury: can the consequences be stopped?, CMAJ 178, 1163-1170.

8. Risdall, J. E., and Menon, D. K. (2011) Traumatic brain injury, Phil. Trans. R. Soc. B: Biol. Sci. 366, 241250. 
9. Langlois, J. A., Rutland-Brown, W., and Thomas, K. E. Emergency department visits, hospitalizations, and deaths. Traumatic brain injury in the United States; US Department of Health and Human Services: Atlanta, 2006; 1-33.

10. Werner, C., and Engelhard, K. (2007) Pathophysiology of traumatic brain injury, Br. J. Anaesth. 99, 4-9.

11. Xiong, Y., Mahmood, A., and Chopp, M. (2009) Emerging treatments for traumatic brain injury, Expert Opin. Emerg. Drugs 14, 67-84.

12. Draper, K., and Ponsford, J. (2009) Long-term outcome following traumatic brain injury: A comparison of subjective reports by those injured and their relatives, Neuropsychol. Rehabil. $19,645-661$.

13. Shlosberg, D., Benifla, M., Kaufer, D., and Friedman, A. (2010) Blood-brain barrier breakdown as a therapeutic target in traumatic brain injury, Nat. Rev.Neurol. 6, 393.

14. Walker, K., and Tesco, G. (2013) Molecular mechanisms of cognitive dysfunction following traumatic brain injury, Front. Aging Neurosci. 5, 29.

15. Johnson, V. E., Stewart, W., and Smith, D. H. (2013) Axonal pathology in traumatic brain injury, Exp. Neurol. 246, 35-43.

16. Povlishock, J. T. (1993) Pathobiology of traumatically induced axonal injury in animals and man, Ann. Emergency Med. 22, 980-986.

17. Przekwas, A., Somayaji, M. R., and Gupta, R. K. (2016) Synaptic Mechanisms of Blast-Induced Brain Injury, Front. Neurol. 7, 2-2.

18. Johnstone, V. P. A., Yan, E. B., Alwis, D. S., and Rajan, R. (2013) Cortical Hypoexcitation Defines Neuronal Responses in the Immediate Aftermath of Traumatic Brain Injury, PLoS One 8, e63454.

19. Allitt, B. J., Iva, P., Yan, E. B., and Rajan, R. (2016) Hypo-excitation across all cortical laminae defines intermediate stages of cortical neuronal dysfunction in diffuse traumatic brain injury, Neuroscience 334, 290-308.

20. Chwiej, J., Sarapata, A., Janeczko, K., Stegowski, Z., Appel, K., and Setkowicz, Z. (2011) X-ray fluorescence analysis of long-term changes in the levels and distributions of trace elements in the rat brain following mechanical injury, J. Biol. Inorg. Chem. 16, 275-283.

21. Ward, R. J., Zucca, F. A., Duyn, J. H., Crichton, R. R., and Zecca, L. (2014) The role of iron in brain ageing and neurodegenerative disorders, Lancet Neurol. 13, 1045-1060.

22. Surowka, A. D., Wrobel, P., Adamek, D., Radwanska, E., and Szczerbowska-Boruchowska, M. (2015) Synchrotron radiation based $X$-ray fluorescence shows changes in the elemental composition of the human substantia nigra in aged brains, Metallomics 7, 1522-1531.

23. Surowka, A. D., Töpperwien, M., Bernhardt, M., Nicolas, J. D., Osterhoff, M., Salditt, T., Adamek, D., and Szczerbowska-Boruchowska, M. (2016) Combined in-situ imaging of structural organization and elemental composition of substantia nigra neurons in the elderly, Talanta 161, 368-376.

24. Hackett, M. J., Hollings, A., Majimbi, M., Brook, E., Cochran, B., Giles, C., Lam, V., Nesbit, M., Rye, K.-A., Mamo, J. C. L., and Takechi, R. (2019) Multimodal Imaging Analyses of Brain Hippocampal Formation Reveal Reduced Cu and Lipid Content and Increased Lactate Content in Non-Insulin-Dependent Diabetic Mice, ACS Chem. Neurosci. 10, 2533-2540.

25. Fimognari, N., Hollings, A., Lam, V., Tidy, R. J., Kewish, C. M., Albrecht, M. A., Takechi, R., Mamo, J. C. L., and Hackett, M. J. (2018) Biospectroscopic Imaging Provides Evidence of Hippocampal Zn Deficiency and Decreased Lipid Unsaturation in an Accelerated Aging Mouse Model, ACS Chem. Neurosci. 9, 2774-2785.

26. Hackett, M. J., Hollings, A., Caine, S., Bewer, B. E., Alaverdashvili, M., Takechi, R., Mamo, J. C. L., Jones, M. W. M., de Jonge, M. D., Paterson, P. G., Pickering, I. J., and George, G. N. (2019) Elemental characterisation of the pyramidal neuron layer within the rat and mouse hippocampus, Metallomics 11, 151-165. 
27. Pushie, M., Kelly, M., and Hackett, M. (2018) Direct label-free imaging of brain tissue using synchrotron light: a review of new spectroscopic tools for the modern neuroscientist, Analyst 143, 3761-3774.

28. Lins, B. R., Pushie, J. M., Jones, M., Howard, D. L., Howland, J. G., and Hackett, M. J. (2016) Mapping Alterations to the Endogenous Elemental Distribution within the Lateral Ventricles and Choroid Plexus in Brain Disorders Using X-Ray Fluorescence Imaging, PLoS One 11, e0158152.

29. Caine, S., Hackett, M. J., Hou, H., Kumar, S., Maley, J., Ivanishvili, Z., Suen, B., Szmigielski, A., Jiang, Z., Sylvain, N. J., Nichol, H., and Kelly, M. E. (2016) A novel multi-modal platform to image molecular and elemental alterations in ischemic stroke, Neurobiol. Dis. 91, 132-142.

30. Bishop, D. P., Cole, N., Zhang, T., Doble, P. A., and Hare, D. J. (2018) A guide to integrating immunohistochemistry and chemical imaging, Chem. Soc. Rev. 47, 3770-3787.

31. Hare, D. J., Jones, M. W. M., Wimmer, V. C., Jenkins, N. L., de Jonge, M. D., Bush, A. I., and McColl, G. (2016) High-resolution complementary chemical imaging of bio-elements in Caenorhabditis elegans, Metallomics 8, 156-160.

32. Hackett, M. J., Aitken, J. B., El-Assaad, F., McQuillan, J. A., Carter, E. A., Ball, H. J., Tobin, M. J., Paterson, D., de Jonge, M. D., Siegele, R., Cohen, D. D., Vogt, S., Grau, G. E., Hunt, N. H., and Lay, P. A. (2015) Mechanisms of murine cerebral malaria: Multimodal imaging of altered cerebral metabolism and protein oxidation at hemorrhage sites, Sci. Adv. 1, e1500911.

33. Miller, L. M., Wang, Q., Telivala, T. P., Smith, R. J., Lanzirotti, A., and Miklossy, J. (2006) Synchrotron-based infrared and X-ray imaging shows focalized accumulation of $\mathrm{Cu}$ and $\mathrm{Zn}$ colocalized with beta-amyloid deposits in Alzheimer's disease, J. Struct. Biol. 155, 30-37.

34. Pushie, M. J., Crawford, A. M., Sylvain, N. J., Hou, H., Hackett, M. J., George, G. N., and Kelly, M. E. (2018) Revealing the Penumbra through Imaging Elemental Markers of Cellular Metabolism in an Ischemic Stroke Model, ACS Chem. Neurosci. 5, 886-893.

35. Hackett, M. J., DeSouza, M., Caine, S., Bewer, B., Nichol, H., Paterson, P. G., and Colbourne, F. (2015) A New Method To Image Heme-Fe, Total Fe, and Aggregated Protein Levels after Intracerebral Hemorrhage, ACS Chem. Neurosci. 6, 761-770.

36. Williamson, M. R., Dietrich, K., Hackett, M. J., Caine, S., Nadeau, C. A., Aziz, J. R., Nichol, H., Paterson, P. G., and Colbourne, F. (2016) Rehabilitation Augments Hematoma Clearance and Attenuates Oxidative Injury and Ion Dyshomeostasis After Brain Hemorrhage, Stroke 48, 195203.

37. Summers, K. L., Fimognari, N., Hollings, A., Kiernan, M., Lam, V., Tidy, R. J., Paterson, D., Tobin, M. J., Takechi, R., George, G. N., Pickering, I. J., Mamo, J. C., Harris, H. H., and Hackett, M. J. (2017) A Multimodal Spectroscopic Imaging Method To Characterize the Metal and Macromolecular Content of Proteinaceous Aggregates ("Amyloid Plaques"), Biochemistry 56, 4107-4116.

38. Kastyak, M. Z., Szczerbowska-Boruchowska, M., Adamek, D., Tomik, B., Lankosz, M., and Gough, K. M. (2010) Pigmented creatine deposits in Amyotrophic Lateral Sclerosis central nervous system tissues identified by synchrotron Fourier Transform Infrared microspectroscopy and X-ray fluorescence spectromicroscopy, Neuroscience 166, 1119-1128.

39. Xiong, Y., Mahmood, A., and Chopp, M. (2013) Animal models of traumatic brain injury, Nat. Rev. Neurosci. 14, 128-142.

40. Anthony, M., Montasser, A. A.-E. F., Wimer van den, B., Campbell, J., Kita, H., and Demetriadou, K. (1994) A new model of diffuse brain injury in rats, J. Neurosurg. 80, 291-300.

41. Yan, E. B., Johnstone, V. P. A., Alwis, D. S., Morganti-Kossmann, M. C., and Rajan, R. (2013) Characterising effects of impact velocity on brain and behaviour in a model of diffuse traumatic axonal injury, Neuroscience 248, 17-29.

42. Gupta, R., and Przekwas, A. (2013) Mathematical Models of Blast-Induced TBI: Current Status, Challenges, and Prospects, Front. Neurol. 4, 59.

43. Carron, S. F., Yan, E. B., Alwis, D. S., and Rajan, R. (2016) Differential susceptibility of cortical and subcortical inhibitory neurons and astrocytes in the long term following diffuse traumatic brain injury, J. Comp. Neurol. 524, 3530-3560. 
44. Li, Y., Zhang, L., Kallakuri, S., Zhou, R., and Cavanaugh, J. M. (2011) Quantitative relationship between axonal injury and mechanical response in a rodent head impact acceleration model, J. Neurotrauma 28, 1767-1782.

45. Pevsner, A., and Diem, M. (2003) IR spectroscopic studies of major cellular components. III. Hydration of protein, nucleic acid, and phospholipid films, Biopolymers 72, 282-289.

46. Homayoun, P., Rodriguez de Turco, E. B., Parkins, N. E., Lane, D. C., Soblosky, J., Carey, M. E., and Bazan, N. G. (1997) Delayed Phospholipid Degradation in Rat Brain After Traumatic Brain Injury, J. Neurochem. 69, 199-205.

47. Marklund, N., Salci, K., Lewén, A., and Hillered, L. (1997) Glycerol as a marker for post-traumatic membrane phospholipid degradation in rat brain, Neuroreport 8, 1457-1460.

48. Hillered, L., Valtysson, J., Enblad, P., and Persson, L. (1998) Interstitial glycerol as a marker for membrane phospholipid degradation in the acutely injured human brain, J. Neurol. Neurosurg. Psychiatry 64, 486-491.

49. Lewén, A., and Hillered, L. (1998) Involvement of reactive oxygen species in membrane phospholipid breakdown and energy perturbation after traumatic brain injury in the rat, $J$. Neurotrauma 15, 521-530.

50. Marion, C. M., Radomski, K. L., Cramer, N. P., Galdzicki, Z., and Armstrong, R. C. (2018) Experimental Traumatic Brain Injury Identifies Distinct Early and Late Phase Axonal Conduction Deficits of White Matter Pathophysiology, and Reveals Intervening Recovery, J. Neurosci. 38, 8723-8736.

51. Mierzwa, A. J., Sullivan, G. M., Armstrong, R. C., Marion, C. M., and McDaniel, D. P. (2015) Components of Myelin Damage and Repair in the Progression of White Matter Pathology After Mild Traumatic Brain Injury, J. Neuropathol. Exp. Neurol. 74, 218-232.

52. Sullivan, G. M., Armstrong, R. C., Mierzwa, A. J., Tang, H., Selwyn, R., Kijpaisalratana, N., Song, S.K., and Wang, Y. (2013) Oligodendrocyte Lineage and Subventricular Zone Response to Traumatic Axonal Injury in the Corpus Callosum, J. Neuropathol. Exp. Neurol. 72, 1106-1125.

53. Payne, S. C., Bartlett, C. A., Harvey, A. R., Dunlop, S. A., and Fitzgerald, M. (2011) Chronic Swelling and Abnormal Myelination during Secondary Degeneration after Partial Injury to a Central Nervous System Tract, J. Neurotrauma 28, 1077-1088.

54. Taib, T., Leconte, C., Van Steenwinckel, J., Cho, A. H., Palmier, B., Torsello, E., Lai Kuen, R., Onyeomah, S., Ecomard, K., Benedetto, C., Coqueran, B., Novak, A.-C., Deou, E., Plotkine, M., Gressens, P., Marchand-Leroux, C., and Besson, V. C. (2017) Neuroinflammation, myelin and behavior: Temporal patterns following mild traumatic brain injury in mice, PLoS One 12, e0184811-e0184811.

55. Barabas, M. E., Mattson, E. C., Aboualizadeh, E., Hirschmugl, C. J., and Stucky, C. L. (2014) Chemical Structure and Morphology of Dorsal Root Ganglion Neurons from Naive and Inflamed Mice, J. Biol. Chem. 289, 34241-34249.

56. Chwiej, J. G., Ciesielka, S. W., Skoczen, A. K., Janeczko, K. J., Sandt, C., Planeta, K. L., and Setkowicz, Z. K. (2019) Biochemical Changes Indicate Developmental Stage in the Hippocampal Formation, ACS Chem. Neurosci. 10, 628-635.

57. Tsang, D., Tsang, Y. S., Ho, W. K. K., and Wong, R. N. S. (1997) Myelin Basic Protein Is a Zinc-Binding Protein in Brain: Possible Role in Myelin Compaction, Neurochem. Res. 22, 811-819.

58. Hansen, A. J. (1985) Effect of Anoxia on Ion Distribution in the Brain, Physiol. Rev. 65, 101-147.

59. Hansen, A. J., and Zeuthen, T. (1981) Extracellular ion concentrations during spreading depression and ischemia in the rat brain cortex, Acta Physiol. 113, 437-445.

60. Malek, S. A., Coderre, E., and Stys, P. K. (2003) Aberrant Chloride Transport Contributes to Anoxic/Ischemic White Matter Injury, J. Neurosci. 23, 3826-3836.

61. Stys, P. K. (1998) Anoxic and Ischemic Injury of Myelinated Axons in CNS White Matter: From Mechanistic Concepts to Therapeutics, J. Cereb. Blood Flow Metab. 18, 2-25. 
62. Hackett, M. J., Britz, C. J., Nichol, H., Paterson, P. G., Pickering, I. J., and George, G. N. (2015) In situ Bio-Spectroscopic Investigation of Rapid Ischemic and Post-mortem Induced Biochemical Alterations in the Rat Brain, ACS Chem. Neurosci. 6, 226-238.

63. Hackett, M. J., McQuillan, J. A., El-Assaad, F., Aitken, J. B., Levina, A., Cohen, D. D., Siegele, R., Carter, E. A., Grau, G. E., Hunt, N. H., and Lay, P. A. (2011) Chemical alterations to murine brain tissue induced by formalin fixation: implications for biospectroscopic imaging and mapping studies of disease pathogenesis, Analyst 136, 2941-2952.

64. Gomez-Fernandez, J. C., and Villalain, J. (1998) The use of FT-IR for quantitative studies of the apparent pKa of lipid carboxyl groups and the dehydration degree of the phosphate group of phospholipids, Chem. Phys. Lipids 96, 41-52.

65. Paterson, D., De Jonge, M., Howard, D., Lewis, W., McKinlay, J., Starritt, A., Kusel, M., Ryan, C., Kirkham, R., and Moorhead, G. (2011) The X-ray Fluorescence Microscopy Beamline at the Australian Synchrotron, AIP Conf. Proc. 1365, 219-222.

66. Ryan, C., Siddons, D., Kirkham, R., Li, Z., de Jonge, M., Paterson, D., Kuczewski, A., Howard, D., Dunn, P., and Falkenberg, G. (2014) MAIA X-ray fluorescence imaging: capturing detail in complex natural samples, J. Phys. Conf. Ser. 499, 012002.

67. Grubman, A., James, S., James, J., Duncan, C., Volitakis, I., Hickey, J., Crouch, P., Donnelly, P., Kanninen, K., and Liddell, J. (2014) X-ray fluorescence imaging reveals subcellular biometal disturbances in a childhood neurodegenerative disorder, Chem. Sci. 5, 2503-2516.

68. Alwis, D. S., Johnstone, V., Yan, E., and Rajan, R. (2013) Diffuse traumatic brain injury and the sensory brain, Clin. Exp. Pharmacol. Physiol. 40, 473-483.

69. Hellewell, S. C., Yan, E. B., Agyapomaa, D. A., Bye, N., and Morganti-Kossmann, M. C. (2010) Post Traumatic Hypoxia Exacerbates Brain Tissue Damage: Analysis of Axonal Injury and Glial Responses, J. Neurotrauma 27, 1997-2010.

70. Carron, S. F., Yan, E. B., Alwis, D. S., and Rajan, R. (2016) Differential susceptibility of cortical and subcortical inhibitory neurons and astrocytes in the long term following diffuse traumatic brain injury, J. Comp. Neurol. 524, 3530-3560.

71. Johnson, V. E., Stewart, J. E., Begbie, F. D., Trojanowski, J. Q., Smith, D. H., and Stewart, W. (2013) Inflammation and white matter degeneration persist for years after a single traumatic brain injury, Brain : a journal of neurology 136, 28-42.

72. Yan, Y., Zhang, L., Kallakuri, S., Zhou, R., Cavanaugh, J.M (2011) Quantitative relationship between axonal injury and mechanical response in a rodent head impact acceleration model, $J$. Neurotrauma 28, 1767-1782. 